\title{
HIV Hospital Admissions Attributable to Specific Opportunistic Infections and Factors Associated with Them at a Botswana Referral Hospital
}

\author{
John Thato Tlhakanelo', Jose Gaby Tshikuka',2, Mooketsi Molefi, ${ }^{1,3}$, \\ Mgaywa Gilbert Mjungu Damas Magafu1, Tiny Masupe1, \\ Reginald Blessing Matchaba-Hove ${ }^{4}$ \\ ${ }^{1}$ Department of Family Medicine and Public Health, Faculty of Medicine, University of Botswana, Gaborone, \\ Botswana \\ ${ }^{2}$ Department of Health Sciences, National Pedagogic University, Kinshasa, DRC \\ ${ }^{3}$ School of Health Systems and Public Health, Faculty of Health Sciences, University of Pretoria, South Africa \\ ${ }^{4}$ School of Public Health, Faculty of Health Sciences, University of Botswana, Gaborone, Botswana \\ Email: jtshikuka@hotmail.com
}

Received 2 July 2015; accepted 28 August 2015; published 31 August 2015

Copyright (C) 2015 by authors and Scientific Research Publishing Inc.

This work is licensed under the Creative Commons Attribution International License (CC BY).

http://creativecommons.org/licenses/by/4.0/

(c) (i) Open Access

\section{Abstract}

Hospital admissions among people living with HIV (PLWH) in Botswana are high. Opportunistic infections (OIs) are responsible for most of these admissions. Although leading OIs causing these admissions have been identified in the region, their correlates are poorly understood. This study aimed to: 1) evaluate major OIs responsible for admissions among HIV patients at Princess Marina Hospital (PMH) in Botswana; 2) estimate the proportion and identify the most frequent admissions attributable to specific OIs; 3 ) characterize major correlates of admissions attributable to each specific OIs and identify populations most at risk as a base for effective policy and resource orientation. HIV infected patients were randomly selected from hospital record lists. Biomedical, sociodemographic and economic data were collected from the records and from face-to-face patient interviews and analyzed. Tuberculosis was the most important $\mathrm{OI}$ responsible for 234.6 per 1000 HIV admissions. Cryptococcal meningitis accounted for 162.0 per 1000 admissions. Patients with a CD4-cell count $<350 / \mu \mathrm{L}$ and males were more likely to be admitted for both tuberculosis and cryptococcal meningitis compared to those with a CD4-cell count $>350 / \mu \mathrm{L}$ and females. The risk of admission due to cryptococcal meningitis was also high among patients with low socioeconomic status (SES). Females were more at risk for Cryptosporidium, Bacterial pneumonia (BP), Pneumocystis Carinii Pneumonia (PCP), Herpes and candidiasis-specific admissions than male and, patients not on co-trimoxazole were more likely to be admitted than those on co-trimoxazole. 
Comprehensive implementation strategies to address OIs among PLWH are needed. To be effective, such strategies should address not only biomedical factors but should also focus on PLWH's SES.

\section{Keywords}

\section{Botswana, People Living with HIV, Opportunistic Infections, Hospital Admissions}

\section{Introduction}

The challenge of controlling and preventing opportunistic infections (OIs) among people living with HIV (PLWH) is brought about by numerous factors. Some of these factors are biomedical like CD4-cell count, case management algorithms e.g. combination antiretroviral therapy (cART) regimen and adherence. Likewise, factors triggering hospital admission for OIs vary widely and may relate to: 1) patients' underlying health conditions e.g. kidney failure, diabetes, hypertension; 2) geographical location; 3) living conditions e.g. poverty; 4) socio-demographic factors e.g. age and gender; and 5) behavioral characteristics e.g. alcoholism and substance abuse. All these factors and their interactions need to be well studied and be understood to better comprehend circumstances that prompt OI hospital admissions in PLWH [1]. Such information could help in the designing of effective programs to control and prevent morbidity due to OIs among PLWH.

Given the existing variability in factors associated with OIs [2]-[4], the burden or proportion of specific types of the OIs responsible for hospital admissions also need to be elucidated at country or regional levels. In the Americas, for instance, pneumococcal carinii pneumonia (PCP) is listed as the predominant OI among HIV admissions [3]-[6]. Yet in some African and other low income countries cryptococcal meningitis has been cited as the major OI among HIV patients [3]-[6]. Research works from Asia on the other hand identified oroesophageal candidiasis as the most common OI associated with almost $60 \%$ of hospital admissions [7]. This setting had $47 \%$ of AIDS patients co-infected with cryptosporidium but only 7\% of admissions due to PCP [7]. These differences not only support regional variability of major OIs among PLWH, but also point to the impact of environmental and general living conditions such as consumption of unsafe drinking water. These figures show that there is a pressing need for suitable approaches in clinical management, prevention and control of HIV-related OIs at regional and country levels. Each region or country needs to know the factors associated with specific OIs causing admissions among her PLWH so as to inform interventions.

Unfortunately, decades after launching HIV intervention programs in Southern Africa, this kind of information is still missing in many countries including Botswana. In these African settings, the burden of OIs among HIV patients is presumably high [8]. A recent study conducted by Thato Tlhakanelo and co-workers has showed that 520 per 1000 admissions of PLWH at a local referral hospital in Gaborone were attributable to OIs namely tuberculosis, cryptococcal meningitis, cryptosporidium, bacterial pneumonia, PCP, herpes and candidiasis [8]. The study did not, however, attempt to investigate correlates of each of these specific OI-related admissions. Such an attempt would be of interest not only to Botswana where $19.3 \%$ of the population live with HIV and clinically featured by various types of OIs, but also to the entire region in designing suitable control and prevention strategies for OIs. Ministries of health, stakeholders and health organizations certainly need this information to adjust their policies and priorities accordingly.

We therefore aim in this work: 1) to estimate the proportion of admissions attributable to specific OIs among PLWH and identify the most frequent ones; 2) to characterize major correlates of admission attributable to each specific OI; and 3) identify groups of PLWH most at risk. The findings of the study will be used to inform effective policy and decision-making to improve the quality of life of PLWH.

\section{Methods}

\subsection{Study Design}

Details on the study design, site and methodology are as outlined by Tlhakanelo and co-workers [8]. In summary, this was a cross-sectional study conducted at Princess Marina Hospital (PMH), the main referral hospital in 
Botswana. The hospital is the busiest in the country with a bed capacity of 567 and staffed by 200 doctors and over 400 registered nurses.

\subsection{Sample Size and Sampling Method}

The sample size was estimated to be 185 participants following the approach used by Arya and colleagues for sample size estimation in prevalence studies [9]. HIV positive in-patients were identified from hospital admission lists every other day. The lists were used as the sampling frame from which study participants were selected using computer-generated random numbers. Selected numbers falling to HIV-negative patients or to those with an unknown HIV status were replaced by new numbers so as to enroll only HIV-infected patients in the study. The data collection process lasted 12 weeks from April to June 2014. Prospective participants were approached in the hospital wards and written consent to participate in the study was obtained from each participant. Participants' medical records were then reviewed and biomedical and socio-demographic data were collected. Faceto-face interviews with patients were conducted and further socio-demographic and economic data missing from their records were collected. Patients aged less than 18 years of age were excluded from the study. Also excluded from the study were pregnant women, patients admitted in the high dependency cubicles as they were considered severely ill, HIV-negative patients or those with unknown HIV status and patients in private wards. To ensure high quality of data and reduce biases, data collectors were trained and pre-tested as well as the data collecting tool. Necessary amendments were made in the instrument and the best data collectors selected for the study. In addition regular supervision of data collection was carried out by the study coordination team.

\subsection{Data Analysis}

Data were entered into the computer using Microsoft Excel (Redmond, WA). The dataset was then imported into IBM SPSS version 21 (Chicago, IL) which was used for analysis. Frequency distributions (\%) of patients’ by age group, gender and cause of admission were computed. Proportions of admissions attributable to aggregated OIs were obtained by computing the number of aggregated admissions due to any form of OIs over the total HIV-admission times 1000. The proportion of admissions attributable to specific OIs among HIV patients were estimated by computing the number of all cases of admissions due specifically to: 1) tuberculosis; 2) cryptococcal meningitis; 3) cryptosporidium, bacterial pneumonia, PCP, herpes and candidiasis over the total HIV-admissions times 1000 and comparisons between groups were made.

Crude associations of admissions attributable to each specific OI with participants' biomedical, socio-demographic and economic characteristics were investigated through bivariate analyses and unadjusted odds ratios (UOR) and their 95\% confidence intervals (CIs) were estimated. All potential correlates of OIs admissions were further investigated in logistic regression sub-models. Adjusted odds ratios (AOR) and their 95\% CIs were computed for every factor retained in main sub-models. Variance inflation factor diagnostic was performed for redundancy and multicollinearity of covariates. How well the model fitted with the data was estimated using the Hosmer-Lemeshow test of goodness-of-fit. The level of significance in the main sub-models was set at $\mathrm{p}<0.05$. Comparisons among sub-groups were made using Chi-square analysis with Fisher exact test.

Ethical clearance was obtained from the Office of Research and Development of the University of Botswana and the Ministry of Health. Permission to review patients' records was sought from PMH's Institutional Review Board. Before the interview and record review, each prospective participant was asked for a signed informed consent. To ensure confidentiality, a number was assigned to every participant in lieu of patients' names or any other direct identifier.

\section{Results}

In total, $179 \mathrm{HIV}$-positive patients gave their consent and participated in this study making a response rate of $96.8 \%$. Of these participants, 82 (45.8\%) were males and $97(54.2 \%)$ were females. The mean age ( \pm SE) was $38.2( \pm 0.9)$ years. The youngest patient was 18 years old while the oldest was 67 years old. Seven specific OIs were identified as major causes of admissions among HIV patients namely tuberculosis, cryptococcal meningitis, cryptosporidium, bacterial pneumonia, PCP, herpes and candidiasis. The seven OIs all together were responsible for 520 per $1000 \mathrm{HIV}$ admissions at PMH. Of these, tuberculosis was the most frequent with 234.6 per 1000 HIV admissions, cryptococcal meningitis was responsible for 162.0 per 1000 HIV admissions and cryptospori- 
dium, bacterial pneumonia, PCP, herpes and candidiasis were together responsible for 67.1 per $1000 \mathrm{HIV}$ admissions.

Patients' biomedical, socio-demographic and economic characteristics are presented elsewhere [8]. Results presented in Table 1 for sub-model A for tuberculosis indicate that HIV patients with CD4-cell count less than $350 / \mu \mathrm{L}$ were 3.25 times more at risk for admission with tuberculosis than those who had a CD4-cell count greater than 350/ $\mu \mathrm{L}$ (AOR 3.20; 95\% CI 1.49 - 7.08). Female patients were 38\% less likely to be admitted for tuberculosis compared to male patients (AOR 0.38; 95\% CI 0.18 - 0.79). Sub-model B in Table 2 for cryptococcal meningitis shows almost the same pattern as in Table 1 with the only difference being that the socioeconomic status (SES) of the patients entered in this sub-model as the most important correlate of hospital admission for cryptococcal meningitis. Participants in the low SES category had 4.28 times the risk of being admitted for cryptococcal meningitis compared to participants in the middle SES category (AOR 4.28; 95\% CI 1.61 - 11.3).

Only two variables entered the sub-model $\mathbf{C}$ for cryptosporidium, bacterial pneumonia, PCP, herpes and candidiasis-specific admissions in Table 3. Those who were not on co-trimoxazole had 3.37 times the risk of being admitted to hospital compared to those who were on co-trimoxazole (AOR 3.37; 95\% CI 1.21 - 9.39). Female patients had 3.30 times the risk of being admitted compared to male patients (AOR 3.30; 95\% CI 1.18 - 9.21).

\section{Discussion}

For the first time in Botswana, we are reporting reasons why HIV patients are still being admitted to hospital for specific OIs despite implementation of free cART program for nearly a decade and half. Besides specific major

Table 1. Sub-model $\mathrm{A}^{\psi}$ : Factors independently associated with admission for tuberculosis among people living with HIV at Princess Marina Hospital in Botswana in 2014. Dependent variable: tuberculosis-specific admissions ( $\mathrm{N}=179)$.

\begin{tabular}{|c|c|c|c|c|c|}
\hline \multirow[b]{2}{*}{ Independent variables } & \multirow[b]{2}{*}{ Proportion \% } & \multicolumn{2}{|c|}{ Unadjusted } & \multicolumn{2}{|c|}{ Adjusted } \\
\hline & & OR & $95 \% \mathrm{CI}$ & OR & $95 \% \mathrm{CI}$ \\
\hline \multicolumn{6}{|l|}{ CD4-cell count } \\
\hline $\mathrm{CD} 4<350 / \mu \mathrm{L}$ & 52.5 & $3.31^{\mathrm{a}}$ & $1.54-7.11$ & $3.25^{\mathrm{a}}$ & $1.49-7.08$ \\
\hline $\mathrm{CD} 4>350 / \mu \mathrm{L}$ & 47.5 & 1 & - & 1 & - \\
\hline \multicolumn{6}{|l|}{ Gender } \\
\hline Female & 54.2 & $0.37^{\mathrm{a}}$ & $0.18-0.76$ & $0.38^{\mathrm{a}}$ & $0.18-0.79$ \\
\hline Male & 45.8 & 1 & - & 1 & - \\
\hline
\end{tabular}

${ }^{\mathrm{a}} \mathrm{p}<0.05$; Hosmer-Lemeshow test of goodness-of-fit; ${ }^{\mathrm{p}} \mathrm{p}=0.30$; OR = odds ratio; $\mathrm{CI}=$ confidence interval.

Table 2. Sub-model $\mathrm{B}^{\psi}$ : Factors independently associated with admission for cryptococcal meningitis among people living with HIV at Princess Marina Hospital in Botswana in 2014. Dependent variable: cryptococcal meningitis-specific admissions $(\mathrm{N}=179)$.

\begin{tabular}{|c|c|c|c|c|c|}
\hline \multirow[b]{2}{*}{ Independent variables } & \multirow[b]{2}{*}{ Proportion \% } & \multicolumn{2}{|c|}{ Unadjusted } & \multicolumn{2}{|c|}{ Adjusted } \\
\hline & & OR & $95 \% \mathrm{CI}$ & OR & $95 \% \mathrm{CI}$ \\
\hline \multicolumn{6}{|l|}{ CD4-cell count } \\
\hline $\mathrm{CD} 4<350 / \mu \mathrm{L}$ & 52.5 & $2.76^{\mathrm{a}}$ & $1.15-6.64$ & $3.20^{\mathrm{a}}$ & $1.25-8.06$ \\
\hline $\mathrm{CD} 4>350 / \mu \mathrm{L}$ & 47.5 & 1 & - & 1 & - \\
\hline \multicolumn{6}{|l|}{ Gender } \\
\hline Female & 54.2 & $0.38^{\mathrm{a}}$ & $0.16-0.87$ & $0.42^{\mathrm{a}}$ & $0.18-0.90$ \\
\hline Male & 45.8 & 1 & - & 1 & - \\
\hline \multicolumn{6}{|l|}{ SES } \\
\hline Low SES & 84.4 & $0.26^{\mathrm{a}}$ & $0.10-0.64$ & $4.28^{\mathrm{a}}$ & $1.61-11.3$ \\
\hline Middle SES & 15.6 & 1 & - & 1 & - \\
\hline
\end{tabular}

${ }_{\mathrm{p}}^{\mathrm{a}}<0.05$; Hosmer-Lemeshow test of goodness of fit; ${ }^{\mathrm{p}} \mathrm{p}=0.24$; OR = odds ratio; $\mathrm{CI}=$ confidence interval; $\mathrm{SES}=$ socio-economic status. 
Table 3. Sub-Model $\mathrm{C}^{\psi}$ : Factors independently associated with admission for cryptosporidium, bacterial pneumonia, PCP, herpes and candidiasis among people living with HIV at Princess Marina Hospital in Botswana in 2014. Dependent variable: cryptosporidium, bacterial pneumonia, PCP, herpes and candidiasis-related admissions $(\mathrm{N}=179)$.

\begin{tabular}{cccccc}
\hline & \multicolumn{2}{c}{ Unadjusted } & \multicolumn{2}{c}{ Adjusted } \\
\hline Independent variables & Proportion $\%$ & OR & $95 \% \mathrm{CI}$ & OR & $95 \% \mathrm{CI}$ \\
\hline Co-trimoxazole use & & & & \\
\hline No & 46.4 & $0.39^{\mathrm{a}}$ & $0.14-1.04$ & $3.37^{\mathrm{b}}$ & $1.21-9.39$ \\
Yes & 53.6 & 1 & - & 1 & - \\
Gender & & & & $3.30^{\mathrm{b}}$ & $1.18-9.21$ \\
Female & 54.2 & $2.50^{\mathrm{a}}$ & $0.93-6.72$ & 1 & - \\
\hline
\end{tabular}

${ }^{\mathrm{a}} \mathrm{p}=0.06$ borderline significance; ${ }^{\mathrm{b}} \mathrm{p}<0.05$; Hosmer-Lemeshow test of goodness of fit ${ }^{\psi} \mathrm{p}=0.98$; OR $=$ odds ratio; $\mathrm{CI}=$ confidence interval.

OIs that lead to admission of PLWH at PMH as reported by Tlhakanelo and co-workers [8], we investigated biomedical, socio-demographic and economic correlates of these admissions. Our findings were that: 1) seven OIs namely tuberculosis, cryptococcal meningitis, cryptosporidium, bacterial pneumonia, PCP, herpes and candidiasis were the major contributors to the 520 per 1000 HIV admissions at PMH between April and June 2014; 2) tuberculosis was the most frequent form of OI followed by cryptococcal meningitis, cryptosporidium, bacterial pneumonia, PCP, herpes and candidiasis; 3) patients with a CD4-cell count less than 350/ $\mathrm{L}$ had a greater risk of admission for tuberculosis and cryptococcal meningitis compared to patients with a CD4-cell count greater than $350 / \mu \mathrm{L}$; 4) while female HIV patients were less likely to be admitted for tuberculosis and cryptococcal meningitis compared to males, they were more likely to be admitted than males for cryptosporidium, bacterial pneumonia, PCP, herpes and candidiasis when aggregated; 5) patients not on co-trimoxazole prophylaxis were more at risk of OI admission in this aggregated sub-model compared to patients on co-trimoxazole; and 6) patients with lower SES category were more likely to be admitted for cryptococcal meningitis than patients with middle SES category.

This study has provided evidence that programs aiming at controlling and preventing morbidities among PLWH in the region should primarily focus on OIs particularly tuberculosis, cryptococcal meningitis, cryptosporidium, bacterial pneumonia, PCP, herpes and candidiasis to be effective. Though studies around the world have highlighted various types of OIs as causes of admission among PLWH, their results are compatible with their local context. Results from this study are significant because they are specific to the Botswana context. Of more interest is that all these studies support findings that the CD4-cell count less than $350 / \mu \mathrm{L}$ as a critical correlate of OI-related admissions among PLWH [10]-[12].

It is known that there are some differences between women and men with respect to OIs occurrence. Men for instance are more likely to develop Kaposi's sarcoma compared to women [13] [14]. Cryptococcal meningitis was also found to be more predominant in men than in women [15]. Conversely, women were found more likely to develop herpes infections than men [16]. This explains why in sub-model A and B, whereby the dependent variables were tuberculosis and cryptococcus, women were less likely to be admitted than men, while in the sub-model $\mathrm{C}$ where bacterial pneumonia and herpes are major OIs within the aggregated dependent variable, women are more likely to be admitted than men.

Of even more interest in this work is the association documented between low SES HIV patients and admission due to cryptococcal meningitis. This was unexpected because the Government of Botswana and partners have made HIV management and care including access to cART free for all eligible Batswana irrespective of their SES. In other words, both the rich and poor have equal access to life-saving cART. So the reason why the low SES group is more at risk than the middle SES group remains puzzling. Research works from the Democratic Republic of the Congo (DRC), however, showed similar outcomes [17] [18]. Low SES patients were more at risk of HIV-related morbidity and mortality than high SES patients in DRC yet the Global Fund to fight AIDS, TB and Malaria (GFATM) has made access to cART, HIV management and care free there just as it is in Botswana [17]. The authors concluded from their findings that though the impact of health programs greatly depended on fund availability and sustainability, other factors such as the SES also needed to be addressed to alle- 
viate this difference. SES is a three dimensional factor (education, occupation and income) and the answer might lie in any of these dimensions. Further studies are recommended to address the problem.

The fact that these data are from one single hospital setting, $\mathrm{PMH}$, was discussed as a possible limiting factor in our previous work [8]. Another limitation might be the absence of high SES patients in this study population. However, the significant difference in the outcome between low SES and middle SES groups may suggest that this difference would have been even greater between low SES and high SES groups. Based on this, it is doubtful that factors other than those discussed herein explain these results.

In short, outstanding findings in this study were that: 1) tuberculosis was the most important OIs that led to 234.6 per $1000 \mathrm{HIV}$ admissions at $\mathrm{PMH}$, patients with a CD4-cell count less than 350/ $\mu \mathrm{L}$ and male patients were more likely to be admitted for tuberculosis than patients with a CD4-cell count greater than 350/ $\mu \mathrm{L}$ CD4 and female patients; 2) cryptococcal meningitis led to 162.0 per $1000 \mathrm{HIV}$ admissions at PMH, again patients with a CD4-cell count less than 350/ $\mu \mathrm{L}$ and males were more likely to be admitted for cryptococcosis compared to those with a CD4-cell count greater than 350/ $\mu \mathrm{L}$ CD4 and their female counterparts. Low SES patients were more likely to be admitted than middle SES patients; 3) cryptosporidium, bacterial pneumonia, PCP, herpes, and candidiasis were together responsible for 67.1 per $1000 \mathrm{HIV}$ admissions at $\mathrm{PMH}$, the risk of such admissions were high among patients who were not on co-trimoxazole and among female patients.

\section{Conclusion}

These results are suggestive of the need for comprehensive health strategies to address the burden of OIs among PLWH. To be effective such strategies should not only address biomedical factors of specific disease entities like tuberculosis, cryptococcal meningitis, cryptosporidium, bacterial pneumonia, PCP, herpes, candidiasis, CD4-cell count, cART, gender, prophylactic treatment, etc. but should also focus on PLWH's SES as a determinant of this population's health.

\section{Acknowledgements}

We gratefully acknowledge the technical support received from the Department of Family Medicine and Public Health of the Faculty of Medicine at the University of Botswana. Our special thanks go to PMH management team and the male and female medical ward staff for their assistance during data collection as well as to the patients for their cooperation. Dr Megan Cox’s, (Assistant Program Director, Department of Emergency Medicine, University of Botswana) input is greatly acknowledged. The study was supported by the Health Resources and Services Administration (HRSA) of the US Department of Health and Human Services (HHS) under grant T84HA21125 (Medical Education Partnership Initiative, \$10,000,000). This information or content and conclusions are those of the authors and should not be construed as the official position or policy of, nor should any endorsements be inferred by HRSA, HHS or the US Government.

\section{References}

[1] UNAIDS (2008) Report on the Global AIDS Epidemic. UNAIDS/08.27E/JC1511E, UNAIDS, Geneva.

[2] Corbett, E.L., Churchyard, G.J., Charalabos, S., et al. (2002) Morbidity and Mortality in South African Gold Miners: Impact of Untreated Disease Due to Human Immune Deficiency Virus. Clinical Infectious Diseases, 34, 1251-1258. http://dx.doi.org/10.1086/339540

[3] Yazdanpanah, Y., Chêne, G., Losina, E., et al. (2001) Incidence of Primary Opportunistic Infections in Two Human Immune Deficiency Virus-Infected French Clinical Cohorts. International Journal of Epidemiology, 30, 864-871. http://dx.doi.org/10.1093/ije/30.4.864

[4] Ole-Nguyaine, S., Crump, J.A., Kibiki, G.S., et al. (2004) HIV-Associated Morbidity, Mortality and Diagnostic Testing Opportunities among Inpatients at a Referral Hospital in Northern Tanzania. Annals of Tropical Medicine and Parasitology, 98, 171-179. http://dx.doi.org/10.1179/000349804225003163

[5] Onyemelukwe, G.C. and Musa, B.O. (2002) CD4+ and CD8+ Lymphocytes and Clinical Features of HIV Seropositive Nigerians on Presentation. African Journal of Medicine and Medical Sciences, 31, 229-233.

[6] Krentz, H.B., Kliewer, G. and Gill, M.J. (2005) Changing Mortality Rates and Causes of Death for HIV-Infected Individuals Living in Southern Alberta, Canada from 1984 to 2003. HIV Medicine, 6, 99-106. http://dx.doi.org/10.1111/j.1468-1293.2005.00271.x

[7] Singh, A., Bairy, I. and Shivananda, P.G. (2003) Spectrum of Opportunistic Infections in AIDS Cases. Indian Journal 
of Medical Sciences, 57, 16-21.

[8] Tlhakanelo, J.T., Tshikuka, J.G., Molefi, M., et al. (2015) The Burden of Opportunistic Infections and Associated Exposure Factors among HIV Patients Admitted at Princess Marina Hospital in Botswana. JJAH (submitted).

[9] Arya, R., Antonisamy, B. and Kumar, S. (2012) Sample Size Estimation in Prevalence Studies. Indian Journal of Pediatrics, 79, 1482-1488. http://dx.doi.org/10.1007/s12098-012-0763-3

[10] Ogoina, D., Obiako, R.O., Muktar, H.M., et al. (2012) Morbidity and Mortality Patterns of Hospitalised Adult HIV/ AIDS Patients in the Era of Highly Active Antiretroviral Therapy: A 4-Year Retrospective Review from Zaria, Northern Nigeria. AIDS Research and Treatment, 2012, Article ID: 940580. http://dx.doi.org/10.1155/2012/940580

[11] Grant, A.D., Djomand, G. and De Cock, K.M. (1997) Natural History and Spectrum of Disease in Adults with HIV/AIDS in Africa. AIDS, 11, S43-S54.

[12] Damtie, D., Yismaw, G., Woldeyohannes, D. and Anagaw, B. (2013) Common Opportunistic Infections and Their CD4 Cell Correlates among HIV-Infected Patients Attending at Antiretroviral Therapy Clinic of Gondar University Hospital, Northwest Ethiopia. BMC Research Notes, 6, 534. http://dx.doi.org/10.1186/1756-0500-6-534

[13] Biggar, R.J., Horm, J., Fraumeni, J.F., Greene, M.H. and Goedert, J.J. (1984) Incidence of Kaposi’s Sarcoma and Mycosis Fungoides in the United States Including Puerto Rico. Journal of the National Cancer Institute, 73, 89-94.

[14] Dictor, M. and Attewell, R. (1988) Epidemiology of Kaposi’s Sarcoma in Sweden Prior to the Acquired Immunodeficiency Syndrome. International Journal of Cancer, 42, 346-351. http://dx.doi.org/10.1002/ijc.2910420307

[15] McClelland, E.E., Hobbs, L.M., Rivera, J., et al. (2013) The Role of Host Gender in the Pathogenesis of Cryptococcus neoformans Infections. Public Library of Science ONE, 8, e63632. http://dx.doi.org/10.1371/journal.pone.0063632

[16] Studahl, M., Petzold, M. and Cassel, T. (2013) Disease Burden of Herpes Zoster in Sweden-Predominance in the Elderly and in Women-A Register Based Study. BMC Infectious Diseases, 13, 586. http://dx.doi.org/10.1186/1471-2334-13-586

[17] Tshikuka, J.G., Okenge, L., Lukuka, A., et al. (2014) Severity of Outcomes Associated to Illnesses Funded by GFATM Initiative and Socio Demographic and Economic Factors Associated with HIV/AIDS, TB and Malaria Mortality in Kinshasa Hospitals, DRC. Ethiopian Journal of Health Sciences, 24, 299-306. http://dx.doi.org/10.4314/ejhs.v24i4.4

[18] Tshikuka, J.G., Atua, B.M., Lukuka, A.K., et al. (2012) Severity of Outcomes Associated to Types of HIV Coinfection with TB and Malaria in a Setting Where the Three Pandemics Overlap. Journal of Community Health, 37, 1234-1238. http://dx.doi.org/10.1007/s10900-012-9559-7 